\title{
Conductivity Contrast in SEM Images of Hydrogenated Graphene Grown on SiC
}

\author{
Iwona Jozwik $^{1}$, Jacek M.Baranowski ${ }^{1}$, Kacper Grodecki ${ }^{1}$, Pawel Dabrowski ${ }^{2}$ and Wlodzimierz \\ Strupinski ${ }^{1}$ \\ 1. Institute of Electronic Materials Technology, 133 Wolczynska Str., 01-919 Warsaw, Poland. \\ 2. Department of Solid States Physics, Faculty of Physics and Applied Informatics, University of Lodz, \\ Pomorska 149/153, 90-236 Lodz, Poland
}

Graphene consists of a planar single sheet of $\mathrm{sp}^{2}$-bonded carbon atoms arranged in a two-dimensional (2D) honeycomb lattice. One of methods of obtaining epitaxial graphene is growth on SiC(0001) by CVD (Chemical Vapour Deposition) [1]. In this growth mode the first carbon layer it is not a graphene one, it is attached to $\mathrm{Si}$ atoms of the substrate by $\mathrm{sp}^{3}$ bonds and it is called the buffer layer. The conversion of the buffer layer into graphene may be obtained by hydrogenation process. Hydrogen molecules introduced between the buffer layer and the SiC substrate, break most of the $\mathrm{sp}^{3} \mathrm{Si}-\mathrm{C}$ bonds and the buffer layer converts into graphene lattice [2].

Graphene grown by CVD technique on $4 \mathrm{H}-\mathrm{SiC}(0001)$ substrates and hydrogenated at a high temperatures were subjected to SEM investigation with low energy ( $\sim 0.2 \mathrm{keV}$ ) electrons using Auriga CrossBeam Workstation (Carl Zeiss) equipped with In-lens SE (true SE1) detector and Energy selective Backscattered electrons (EsB, low-loss BSE) detector, both positioned on the optical axis of the Gemini (TM) column. The main aim of the work was to determine the contrast origin of the specified areas of the graphene intercalated by $\mathrm{H}_{2}$. The results obtained by low-kV SEM were supported by LC-AFM (Local Conductivity AFM) technique. The presence of graphene has been confirmed by Raman spectroscopy measurements.

Figure 1 presents the SEM images obtained in the In-Lens (a) and EsB (b) detectors in parallel. Both images show characteristic dark parts in the middle of the terraces, surrounded by brighter zones. The bright lines visible in the images are thermally driven cracks in the graphene (due to the high temperature during hydrogenation process) uncovering the SiC substrate. Assuming that the contrast in the SE image (In-lens) is mostly originating from the conductivity differences, and the image of low-loss BSEs is based on the compositional contrast, the reason of the unquestionable contrast of carbon layers may be explained as follows.

It has been shown that the graphene buffer layer grown of $\mathrm{SiC}(0001)$ is not an uniform one and the area close to step edges are well saturated with carbon atoms [3]. On the other hand, terraces are not so well saturated with carbon atoms and characterize with a larger concentration of defects [3]. The hydrogenation of well saturated regions, close to step edges, will result in breaking $\mathrm{Si}-\mathrm{C} \mathrm{sp}^{3}$ bonds between buffer layer and substrate, and formation of $\mathrm{Si}-\mathrm{H}$ bonds and the p-type conductive graphene layer. However, the defected graphene terrace regions, in addition to formation of $\mathrm{Si}-\mathrm{H}$ bonds underneath of graphene layer, may have defects of a donor character, which will compensate p-type conductivity introduced by the $\mathrm{Si}-\mathrm{H}$ bonds. Therefore, the central regions of terraces may have less conductive character and to be depleted of charge carriers. These subtle phase differences are clearly visible in the EsB images obtained in a very low energy regime, where the yield of low-loss BSEs is strongly influenced by the nature of molecular bonds, thus the presence of defects in graphene may create differences in scattering coefficient. The results have been confirmed by AFM measurements in 
LC-AFM mode. Figure 2b presents AFM image showing local conductivity map of the same sample, with a high-conductivity regions of graphene along step edges and a low-conductivity on terraces.

References:

[1] C Riedl, C Coletti, T Iwasaki, AA Zakharov and U Starke, Phys. Rev. Lett. 103 (2009), p. 246804.

[2] W Strupinski, K Grodecki, A Wysmolek, R Stepniewski, T Szkopek, PE Gaskell, A Gruneis, D Haberer, R Bozek, J Krupka and JM Baranowski, Nano Lett. 11 (2011), p. 1786.

[3] W.Strupinski,K.Grodecki, P.Caban, I.Jozwik-Biała, J.M.Baranowski , Carbon 81, 63-72(2015)

[4] This work was partially supported by the National Centre for Research and Development project GRAFTECH/NCBR/02/19/2012, the European Union Seventh Framework Programme under grant agreement no. 604391Graphene Flagship, the National Center for Research and Development under the GRAFTECH/NCBiR/12/14/2013 GRAF-MAG and the National Centre of Science under the UMO2012/07/N/ST3/03141 Preludium 4 grant.

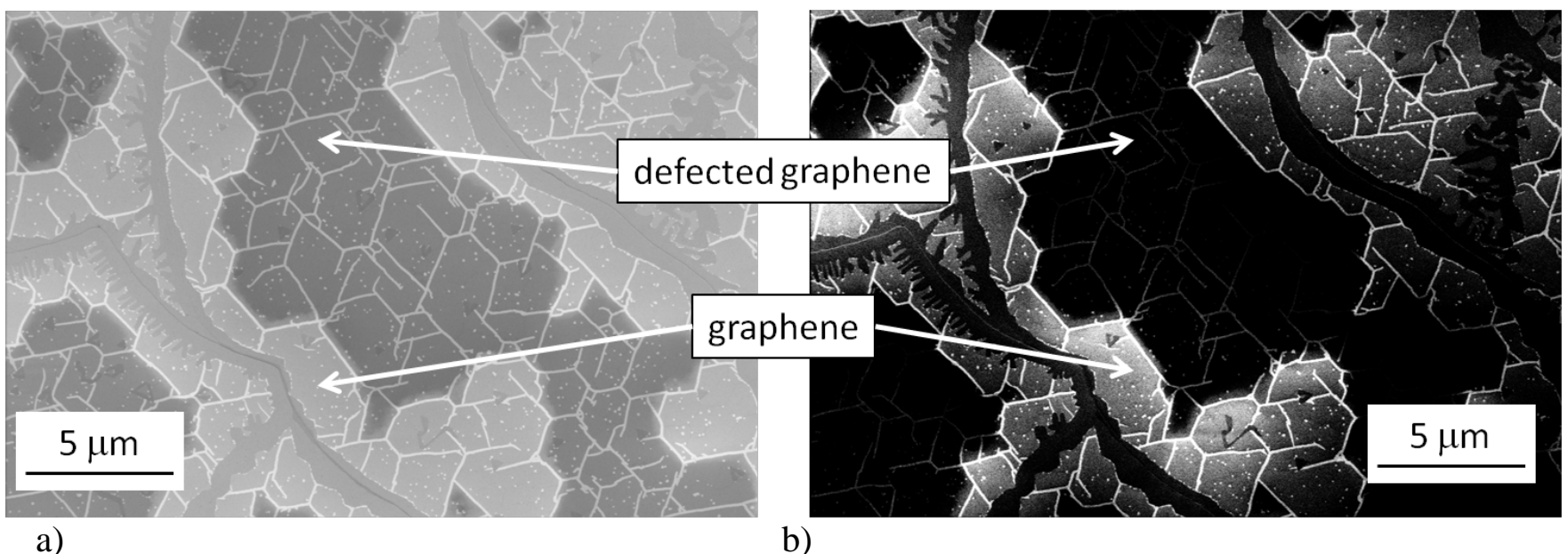

Figure 1. SEM images of CVD-grown graphene intercalated with $\mathrm{H}_{2}$ : a) SE image, b) low-loss BSE image.
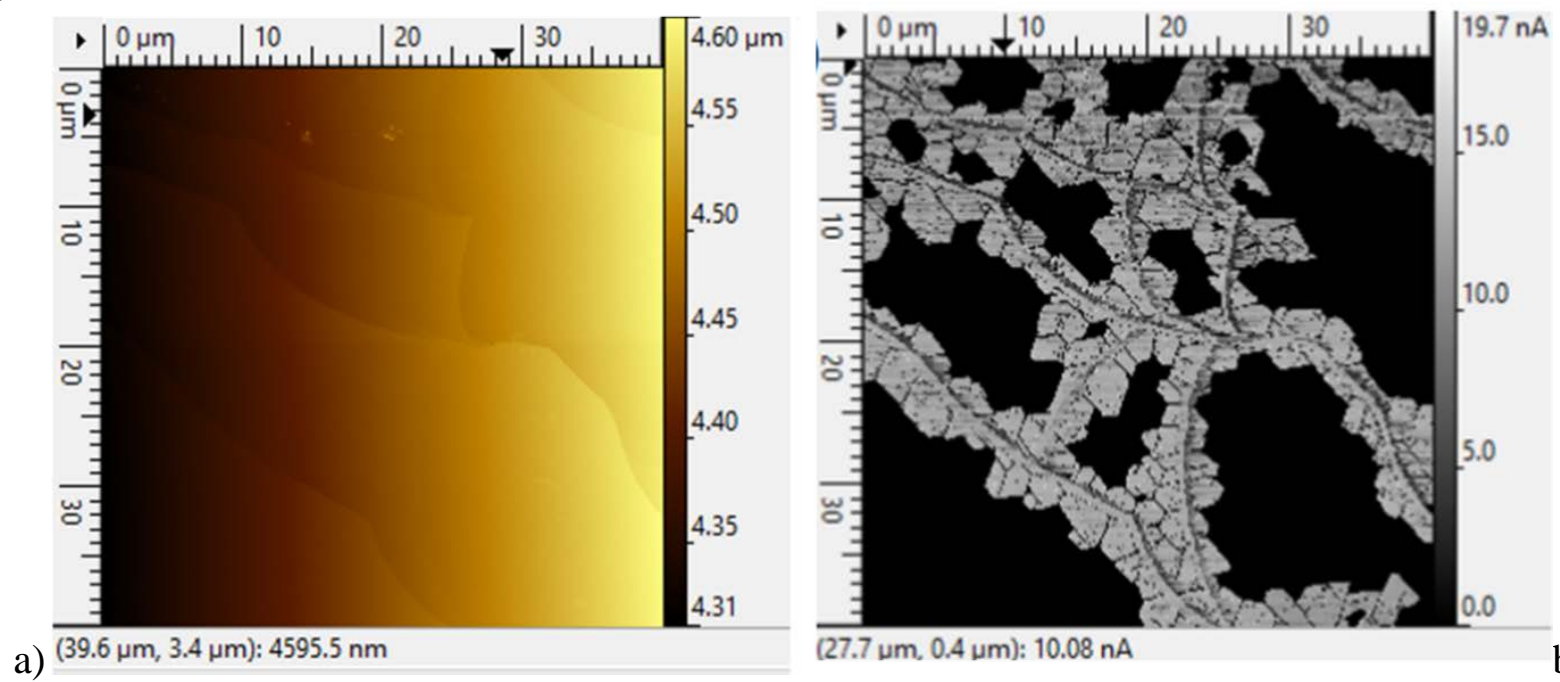

b)

Figure 2. AFM and LC-AFM images: a) topography b) map of local conductivity (dark regions are connected with a low and the bright ones with a high conductivity). 